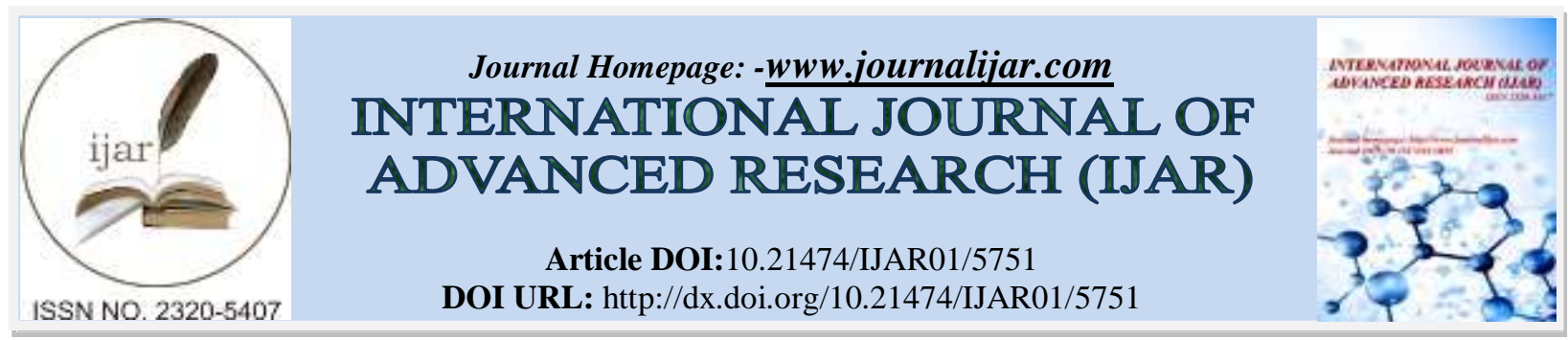

RESEARCH ARTICLE

\title{
SURFACE CHARACTERISTICS OF DENTAL IMPLANTS.
}

Dr.Haritha. T.V ${ }^{1}$,Dr. B.S. Jagadish Pai ${ }^{2}$ and Dr.Nithya R Krishnan ${ }^{3}$.

1. Post Graduate Student,Dept Of Periodontics, CoorgInstitut Of Dental Sciences, Virajpet, Karnataka.

2. HOD And Professor,Dept Of Periodontics, CoorgInstitut Of Dental Sciences, Virajpet, Karnataka.

3. Post Graduate Student DeptOf Periodontics, CoorgInstitut Of Dental Sciences, Virajpet, Karnataka.

\section{Manuscript Info}

Manuscript History

Received: 03 September 2017

Final Accepted: 05 October 2017

Published: November 2017

\begin{abstract}
Over the past decade, several techniques to modify the implant surfaces have been widely studied and developed in an attempt to increase the rate of bone healing and to achieve rapid osteointegration. An appropriately modified titanium surface might certainly by the key factor for achieving a fast and stable implant outcome through optimal osteointegration. The implant-to-bone interface should be able to promote the apposition of new bone by a proper triggering of the biochemical functions. In a similar way, the surface of the implant area in contact with the gingival tissues should enhance the apposition of soft tissues, sealing the way to the ingress, proliferation, and colonization of bacteria from the oral cavity. Besides implant design and surgical technique, surface chemical composition and topography are recognized to be key factors for achieving a fast and durable osteointegration, as well as for implant stability over time.
\end{abstract}

Copy Right, IJAR, 2017,. All rights reserved.

\section{Introduction:-}

The oral rehabilitation of missing teeth by dental implants is one of the most frequently used surgical procedures nowadays. The rate of clinical success of the use of oral implants is widely related to the bone formation at the endoosseous implant surface in contrast with fibrous encapsulation that often leads to loss of the implant and consequent failure of the treatment. This process known as osteointegration was firstly described by Branemark. ${ }^{1}$ Nowadays it is widely accepted by the scientific community that it is an absolute requirement for the successful implant-supported dental prosthesis. Research has resulted in better designs, materials, and more extensive clinical knowledge compared with the early years of implant development. However, the main cause for clinical failure is still insufficient bone formation around the biomaterial, immediately after implantation. ${ }^{2}$ Therefore, improvements are needed in this area as clinicians and patients are pushing for faster healing times.

\section{Repair After Implant Placement:-}

After implant placement into a prepared osteotomy, three stages of repair occur : initial formation of a blood clot occurs through a biochemical activation followed by a cellular activation and finally a cellular response. This events can be subdivided into: hemorrhage into the defect with unspecific protein, adsorption by the dental surface, platelet activation and degranulation, inflammation, recruitment, migration, and adhesion of osteogenic progenitor cells (osteoconduction), osteogenic proliferation, osteogenic differentiation with matrix synthesis, calcification (de novo bone formation), followed by lifelong bone remodeling at the implant surface. ${ }^{3}$ 


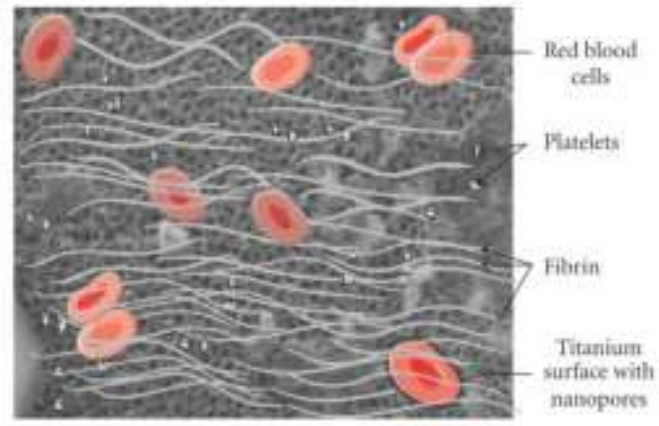

Figure 1:- Interaction of surface of dental implants with blood ${ }^{4}$
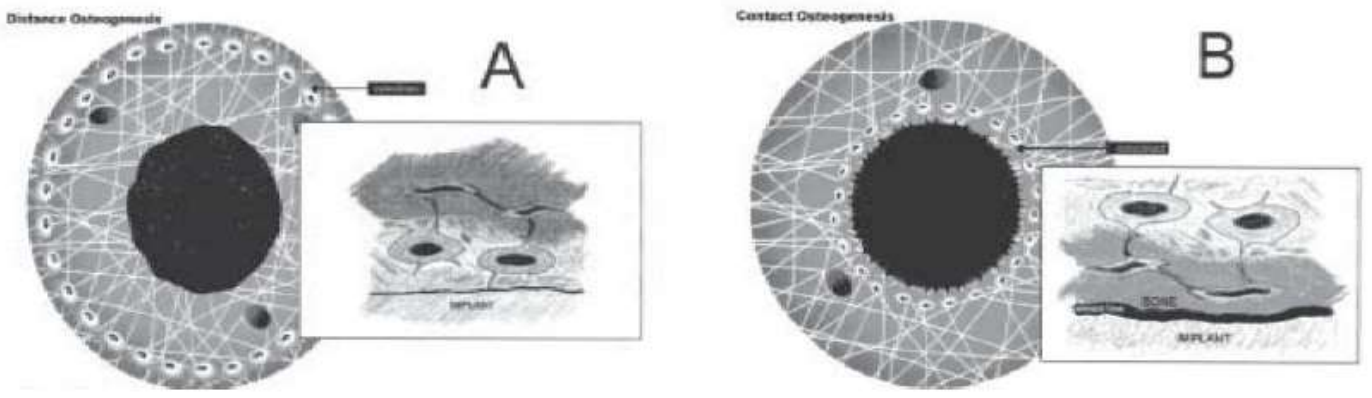

Figure 2:- Drawings to show the initiation of distance osteogenesis (A) and contact osteogenesis (B) where differentiating osteogenic cells line either the old bone or implant surface respectively.

\section{Surface Characteristics:-}

Numerous surface engineering methods have been developed to create featured implant surfaces in order to improve the clinical performance of implants and to guarantee a stable mechanical bone implant interface. ${ }^{5}$ Nowadays, there are several methods to modify the implant surface characteristics with the main objective of improving the biomechanical properties of the implant. Among these techniques, the most common are: Turned surface (machined dental implants), Gritblasting, Grit-blasting and acid-etching, Titanium Plasma Spraying, etc.

\section{1) Turned or machined dental implant surface:}

The first generation of dental implants, termed the turned implants, had a relatively smooth surface after being manufactured, are submitted to cleaning, decontamination and sterilization procedures. ${ }^{1}$ This surfaces are usually and inadequately called "smooth" since scanning electron microscopy analysis showed that they have grooves, ridges and marks derived from tools used for their manufacturing which provides mechanical resistance through bone interlocking. ${ }^{6}$ However, the main disadvantage regarding the morphology of non-treated implants is the fact that osteoblastic cells are prone to grow along the grooves existing on the surface, which in terms of clinical implications means a longer healing time required. ${ }^{7}$

\section{2) Sandblasted surface:}

Increased roughness of an implant could be achieved by blasting the surface by small particles, usually called sandblasting or grit blasting. When the particles hit the implant surface it will create a crater. The surface roughness is hence dependent on the bulk material, the particle material, the particle size, the particle shape, the particle speed and the density of particles. The resulting surface roughness is usually anisotropic consisting of craters and ridges and occasionally particles embedded in the surface. The surface roughness increases with the size of the particles used where $25 \mu \mathrm{m}$ particles blasted surfaces were rougher than the machined surface while smoother than $75 \mu \mathrm{m}$ and $250 \mu \mathrm{m}$ blasted surfaces. Typical Sa values are 0.5-2.0 $\mu \mathrm{m}$. Further, implants blasted with $25 \mu \mathrm{m}$ and $75 \mu \mathrm{m}$ particles show higher removal torque compared to a machined implant surface after 12 weeks of healing in either rabbit tibia or femur. The biological response to blasted implants show a optimal bone response with regards to 
removal torque values and bone implant contact to implants when a roughness of $1.5 \mu \mathrm{m}$ is a achieved. ${ }^{8}$ No ultrastructural studies of the interface between bone and implant surface have been found in the literature for blasted implants.

\section{3) Acid etched surface:}

With acid etching the surface is pitted by removal of grains and grain boundaries of the implant surface, as certain phases and impurities are more sensitive to the etching a selective removal of material is obtained. The resulting roughness is dependent on the bulk material, the surface microstructure, the acid and the soaking time. The surfaces are generally considered minimally rough as the typical Sa values are 0.3-1.0 $\mu \mathrm{m}$. Few analyses of the surface layer have been found, but speculative a titanium hydride layer could form due to the presence of hydrogen ions in the acid. The surface oxide has been found to be a native amorphous titanium oxide with a thickness of around $10 \mathrm{~nm}^{9}$. The bone response to acid etched implants has been compared to machined implants in animal models.

\section{4) Sandblasted and acid etched surface (SLA):}

Commercially available dental implants are usually both blasted by particles and then subsequent etched by acids. This is performed to obtain a dual surface roughness as well as removal of embedded blasting particles. The etching reduces the highest peaks while smaller pits will be created and the average surface roughness will be reduced. By the beginning of the 90s, intensive research had already shown that the sandblasted and acid etched surface had advantages compared to nearly every other type of implant surface, including the titanium plasma spray surface which, until that time, had been the standard for ITI implants. ${ }^{10}$ Typical Sa values for blasted and acid etched implants are 1-2 $\mu \mathrm{m}$. The chemical process of the acid etching will change the surface structure and it has been reported a creation of a titanium hydride layer with a thickness of 1-2 $\mu \mathrm{m}$ intermediate the surface oxide and the bulk metal. Further, by rinsing the SLA implant in a nitrogen atmosphere and storing in saline solution until installation, the amount of carbon contamination could be reduced and improving the hydrophilicity of the implant surface. The result of this procedure is creating a new hydrophilic surface (SLActive) This procedure allows the SLActive to maintain a chemically active surface that conditioned to the human body. Also the anions from the acid could be incorporated in the oxide layer such as fluoride ions if etched in hydrofluoric acid.

\section{5) Anodized surface:}

The anodized surface (TiUnite) is a partial crystalline and phosphate enriched titanium oxide characterized by a microstructured surface with open pores in the low micrometer range.Anodization or anodic oxidization as it's also called is an electrochemical process carried out in an electrolyte. The structural and chemical properties could be tailored by varying different process parameters, such as anode potential, electrolyte composition, temperature and current. Further, depending on the electrolyte composition, different ions could be integrated in the oxide layer, such as phosphorous, calcium and magnesium. ${ }^{9}$ At lower voltages, below the dielectric breakdown limit, a rather constant oxide growth is obtained, while at higher voltages, an increased gas evolution is obtained rendering the surface oxide porous. The crystalline structures of anodized oxides are amorphous with crystalline grains of anatase. TEM analysis of FIB prepared samples showed an amorphous zone between the bone tissue and the implant surface. Furthermore, in the later study, interdiffusion of titanium, phosphorus and calcium between the bone and the coating where intimate bone-implant contact was observed, suggested that chemical bonding also exists within this interface.

\section{6) Laser modified micro- and nano-structured surface:}

Laser is an emerging field for use as a micromachining tool to produce a 3-D structure at micrometer and nanometer level. The technique generates short pulses of light of single wavelength, providing energy focused on one spot. It is rapid, extremely clean, and suitable for the selective modification of surfaces and allows the generation of complex microstructures/ features with high resolution. These advantages make the technique interesting for geometrically complex biomedical implants. The Brånemark BioHelix Implant has surface modified with laser micromachining process to create micro- and nano-structured surface roughness in only the inner part of the thread. The inner part of the thread is believed to be more suitable for bone formation than the outer part. ${ }^{11}$ The laser technique has several advantages, add no chemicals and can be used in routine manufacturing. Only the valley and parts of the flank of the implant threads was laser treated while the remaining part was left as-machined. The idea behind this design is that the flack portion of the implant thread, which might have the higher risk to expose to the microorganism and plaque, is characterised by relatively smooth surface to minimize the incidence of peri-implantitis, whereas the valley part of the implant threads has the rougher surface. 


\section{7) Calcium phosphate coatings on titanium implants:}

Some surface reactive materials have shown the ability to form an interfacial chemical bond with surrounding tissues through a series of biophysical and biochemical reactions, causing 'bioactive fixation' of the implant. ${ }^{12}$ Bioactive materials can be biostable (i.e. synthetic hydroxyapatite) or bioresorbable (i.e. bioactive glasses and glassceramics). Some bioactive ceramics like bioactive glasses of certain compositions have been claimed to have a real chemical bonding ability with soft tissues. Calcium phosphates $(\mathrm{CaP})$ are the most common family of bioceramics well-known for their use in biological application. $\mathrm{CaP}$ in the crystallographic form of apatite is an important mineral constituent of bone. They are considered to be bioactive and osteoconductive. Bioactivity would be due to epitaxial nucleation of carbonated apatite crystals at the surface of ceramic grains. This layer of biological apatite might contain endogenous proteins and might serve as a matrix for osteogenic cell attachment and growth. Different types of methods have been introduced to prepare calcium phosphate coatings on dental implant. These methods can be divided to two groups: physical and chemical methods. Sometimes they can also be called dry and wet methods. Typically physical techniques include plasma spraying deposition, physical vapour deposition, magnetron sputtering deposition, ion beam assisted deposition, pulsed laser deposition, and hot isostatic pressing. Chemical techniques include sol-gel method, biomimetic process, electrochemical deposition, micro-arc oxidation (MAO) and electrophoretic deposition.

\section{Future Prospective In Dental Implant Surfaces:-}

Future development of the next, third generation of dental implants should be based on increased knowledge about the interface biology on cellular and molecular levels. The development of future generations of oral implants for compromised tissue conditions will, most probably, entail tailored modifications of material surfaces. Implant surfaces, selectively, designed for drug and/or cell releases represent promising candidate strategy. Other surface modifications, such as selective ion substitutions of biomimetic surfaces may further improve the biological response to those surfaces.

\section{References:-}

1. Branemark PI, A.R., Breine U, Hansson BO, Lindstrom J, Ohlsson A. ,Intraosseous anchorage of dental prostheses. I. Ex-perimental studies. Scand J PlastReconstrSurg, 1969. 3: p. 81 -1002. Christenson EM, A.K., van den Beucken LJ J Orthop Res, Nano-biomaterial applications in orthopedics. 2007. 25: p. 11-22.

2. Palmquist, A., et al., Titanium oral implants: surface characteristics, interface biology and clinical outcome. J R Soc Interface, 2010. 7 Suppl 5: p. S515-27.

3. Lavenus, S., G. Louarn, and P. Layrolle, Nanotechnology and dental implants. Int J Biomater, 2010. 2010: p. 915327

4. Anselme, K., et al., The relative influence of the topography and chemistry of TiAl6V4 surfaces on osteoblastic cell behaviour. Biomaterials, 2000. 21(15): p. 1567-77. 71

5. AlfarrajAldosari, A., et al., The Influence of implant geometry and surface composition on bone response. Clin Oral Implants Res, 2013.

6. Anil.S, et al., Dental implant surface enhancement and osseointegration. Implant dentistry - a rapidly envolv, 2011.

7. Wennerberg, A. and T. Albrektsson, Effects of titanium surface topography on bone integration: a systematic review. Clin Oral Implants Res, 2009. 20 Suppl 4: p. 172-84.

8. Sul, Y.T., et al., Optimum surface properties of oxidized implants for reinforcement of osseointegration: surface chemistry, oxide thickness, porosity, roughness, and crystal structure. Int J Oral Maxillofac Implants, 2005. 20(3): p. 349-59.

9. Buser, D., et al., Enhanced bone apposition to a chemically modified SLA titanium surface. J Dent Res, 2004. 83(7): p. 529-33.

10. Thomsson M, Esposito M, A retrospective case series evaluating BranemarkBioHelix implants placed in a specialist private practice following conventional procedures. One-year results after placement. Eur J Oral Implantol. 2008 Autumn;1(3):229-34.

11. Cao Larry et al., Bioactive materials.Ceramics International, 1996, 22(6):P. 493-507. 